\title{
Influence of Inclusion Complexation and Skin Microporation on Enhancement of Transdermal Permeation of Raloxifene Hydrochloride
}

\author{
HETAL P. THAKKAR*, H. G. SAVSANI AND P. K. SRIVASTAVA \\ Shri G. H. Patel Pharmacy Building, Faculty of Pharmacy, Maharaja Pratapsinhrao Gaekwad Parisar, The Maharaja Sayajirao \\ University of Baroda, Fatehgunj, Vadodara-390 002, India
}

\author{
Thakkar, et al.: Transdermal Inclusion Complex Gel of Raloxifene
}

\begin{abstract}
The aim of the present investigation was to develop an inclusion complex-based hydrogel for transdermal delivery of raloxifene hydrochloride. Inclusion complexation was tried using two types of cyclodextrins, $\beta$-cyclodextrin and hydroxypropyl- $\beta$-cyclodextrin. Kneading, co-precipitation, solvent evaporation and freeze drying were the methods explored for preparing inclusion complexes. The prepared complexes were characterized using differential scanning calorimetry, Fourier-transform infrared spectroscopy, X-ray powder diffraction and both in vitro and ex vivo drug release studies. Kneading method was found to be the most suitable for preparing the inclusion complexes. Phase solubility studies indicated that $\beta$-cyclodextrin gave rise to Bs type of curve while hydroxypropyl- $\beta$-cyclodextrin resulted in Ap type of curve. The stability constants (K1:1) obtained for $\beta$-cyclodextrin and hydroxypropyl- $\beta$-cyclodextrin were 1572 and 2960 , respectively. Complexation efficiency of hydroxypropyl- $\beta$-cyclodextrin was higher than that of $\beta$-cyclodextrin. Differential scanning calorimetry, Fourier-transform infrared spectroscopy and X-ray powder diffraction studies indicated the superiority of hydroxypropyl- $\beta$-cyclodextrin for complexing raloxifene hydrochloride. In vitro and ex vivo studies showed that highest drug release occurred from inclusion complex prepared with hydroxypropyl- $\beta$-cyclodextrin with a ratio of 1:2.5. Histopathology studies revealed that the developed hydrogel was non-irritant and micropores were clearly visible for the microporated skin.
\end{abstract}

Key words: Raloxifene hydrochloride, skin microporation, inclusion complex, transdermal, permeation enhancement

Menopause is a biological process that occurs as a part of ageing in women. One of the key factors of the menopause is oestrogen deficiency. Oestrogen, through its receptors, $\mathrm{ER} \alpha$ and $E R \beta^{[1]}$, plays a key role in modulation of bone density ${ }^{[2]}$. Thus, its deficiency often leads to osteoporosis in post-menopausal women ${ }^{[3]}$, causing a decrease in bone mineral density and an increased risk of fracture. Oestrogen deficiency also leads to the increased risk of breast cancer ${ }^{[4]}$. Hormone replacement therapy (HRT) with oestrogen alone or in combination with progesterone has remained the first choice for the treatment of postmenopausal osteoporosis. However, the prolonged use of HRT further increases the risk of breast cancer ${ }^{[5,6]}$. This dictates the need for an alternative approach for the management of postmenopausal osteoporosis. Out of several available therapies for the treatment of postmenopausal osteoporosis, selective oestrogen receptor modulators are gaining preference over long-term $\mathrm{HRT}^{[7]}$. Raloxifene hydrochloride $(\mathrm{RH})$, [6-hydroxy-2-(4-hydroxyphenyl)benzo-[b]thien3-yl][4-[2-(1-piperidinyl)ethoxy]-phenyl]ethanone hydrochloride, is an important drug of this category. $\mathrm{RH}$ reproduces the beneficial effects of oestrogens on the skeletal systems, without the negative effects oestrogens on breast and endometrium. It is commonly prescribed for the prevention of osteoporosis and also possesses beneficial actions on lipoprotein metabolism, reducing both total cholesterol and low-density lipoprotein. However, the use of RH is severely limited by its poor bioavailability, high inter-individual and intra-

This is an open access article distributed under the terms of the Creative Commons Attribution-NonCommercial-ShareAlike 3.0 License, which allows others to remix, tweak, and build upon the work non-commercially, as long as the author is credited and the new creations are licensed under the identical terms

Accepted 01 November 2018

Revised 12 April 2018

Received 22 March 2017

*Address for correspondence

E-mail: hetal_thakkar11@yahoo.com

January-February 2019

Indian Journal of Pharmaceutical Sciences 
individual variability (30\%) of most pharmacokinetic parameters and several associated adverse events like vasodilation (hot flushes), venous thromboembolism (deep vein thrombosis, pulmonary embolism and retinal vein thrombosis) and leg cramps (idiopathic). It is a biopharmaceutical classification system class II drug with low solubility and high permeability ${ }^{[8]}$. Presently available marketed preparations include film-coated tablets containing $60 \mathrm{mg}$ of $\mathrm{RH}$ per tablet administered orally. Absolute bioavailability of $\mathrm{RH}$ is only $2 \%$ due to its poor water solubility and extensive first-pass metabolism. Novel drug delivery systems such as micro-emulsion ${ }^{[9]}$, self-micro-emulsifying systems $^{[9]}$, solid-lipid nanoparticles ${ }^{[10]}$ and inclusion complexation $^{[11]}$ have been reported to enhance the oral bioavailability of RH. One of the approaches for avoidance of the first pass hepatic metabolism is delivery of drug through alternative routes such as buccal, sublingual, vaginal, nasal, rectal or transdermal. The avoidance of portal circulation and thereby hepatic metabolism leads to the enhancement of bioavailability. The advantages such as patient compliance, possibility of cessation of the treatment if required, avoidance of hepatic first pass metabolism and thereby decrease in metabolite-related side effects make transdermal delivery an attractive route for administration of RH. Transdermal delivery of RH using novel carriers such as nanotransfersomes ${ }^{[12]}$, ethosomes ${ }^{[13]}$ and a patch $^{[14]}$ using various polymers have been reported. Transdermal delivery is limited to potent drugs having favourable partition coefficient and low molecular weight. Stratum corneum (SC) is a principal barrier to the permeation of drug molecules across the skin. RH has extremely poor solubility (aqueous solubility $0.25 \mathrm{mg} / \mathrm{l}$ ) and high lipophilicity as indicated by its $\log$ P value of $5.69^{[15]}$. In order for a drug to cross the SC layer, it needs to be in solubilized state. This necessitates utilization of an approach, which can solubilize RH. Inclusion complexation of drug with cyclodextrin (CD) molecules improves the aqueous solubility of drug required for penetration across viable skin tissues rich in water content. Squeezing out of CD complexes through SC have also been reported to enhance drug permeation ${ }^{[16]}$. Thus, one of the approaches selected for the present study was to prepare the inclusion complex of RH with CD. Our previous work on ethosomes ${ }^{[13]}$ indicated that pre-creation of micropores on the skin using microneedles could significantly increase the transdermal permeation compared to the intact skin. A combination of two or more techniques is usually required to achieve sufficient transdermal permeation.
Application of microneedles, a physical penetration enhancement technique that breaches SC in a painless manner, have shown significant impact on systemic delivery of certain drugs via transdermal route ${ }^{[17]}$. Thus, in the present investigation, a combination of two approaches viz. CD inclusion complexation and use of microneedles was used for enhancement of transdermal permeation of $\mathrm{RH}$.

\section{MATERIALS AND METHODS}

RH was obtained as a gift sample from Aarti Drugs Ltd., Mumbai. $\beta C D$ and hydroxypropyl- $\beta$-CD (HP $\beta C D$ ) were purchased from HiMedia Pvt. Ltd, Mumbai. Dermastamp ${ }^{\circledR}$ was purchased from Coherent Medical Service, Mumbai. All other reagents were of analytical grade.

\section{Phase solubility study ${ }^{[18]}$ :}

About $10 \mathrm{ml}$ of distilled water containing different concentrations of $\beta C D$ and $\mathrm{HP} \beta C D$ (Table 1) were taken into vials and $2 \mathrm{mg}$ of $\mathrm{RH}$ (amount exceeding its solubility) was added to each vial. Mixtures were stirred for two days and allowed to stand for one day at room temperature to attain equilibrium. Content of each vial was filtered using Whatman filter paper and concentration of $\mathrm{RH}$ in filtrate was estimated using a validated UV/Vis spectrophotometric method at $287 \mathrm{~nm}$ absorption maximum. The solubility experiments were conducted in triplicate. A phase solubility diagram was then constructed by plotting the solubility of the substrate $(\mathrm{RH})$ on the $y$-axis and the total molar concentration of ligand (CD) on the $\mathrm{x}$-axis. The complexation efficiency (CE) and the stability constant $\left(\mathrm{K}_{1: 1}\right)$ were calculated from the slope of the graph and the intrinsic solubility $\left(\mathrm{S}_{0}\right)$ of $\mathrm{RH}$ (i.e, solubility in absence of $\mathrm{CD}$ ) by the following Eqn. 1 and 2, respectively: $\mathrm{CE}=$ slope/(1-slope) and $\mathrm{K}_{1: 1}=$ $\mathrm{CE} / \mathrm{S}_{0}=$ slope $/ \mathrm{S}_{0}(1-$ slope $)$.

TABLE 1: PHASE SOLUBILITY STUDIES

\begin{tabular}{lccc}
\hline $\begin{array}{l}\text { Molar ratio } \\
\text { of } \\
\begin{array}{l}\text { RH: } B C D / \\
\text { HPBCD }\end{array}\end{array}$ & $\begin{array}{c}\text { Concentration } \\
\text { of } B C D / H P B C D \\
\text { taken }(\mathrm{mM})\end{array}$ & $\begin{array}{c}\text { Solubility of } \\
\text { RHith } B C D^{*} \\
(\mathrm{mM})\end{array}$ & $\begin{array}{c}\text { Solubility } \\
\text { of RH with } \\
\text { HPBCD* }(\mathrm{mM})\end{array}$ \\
\hline $1: 0$ & 0.0 & $0.134 \pm 0.008$ & $0.134 \pm 0.008$ \\
$1: 0.5$ & 0.195 & $0.081 \pm 0.04$ & $0.153 \pm 0.010$ \\
$1: 1$ & 0.390 & $0.158 \pm 0.02$ & $0.207 \pm 0.012$ \\
$1: 1.5$ & 0.585 & $0.242 \pm 0.031$ & $0.270 \pm 0.018$ \\
$1: 2$ & 0.780 & $0.308 \pm 0.02$ & $0.314 \pm 0.027$ \\
$1: 2.5$ & 0.975 & $0.225 \pm 0.03$ & $0.408 \pm 0.071$ \\
\hline
\end{tabular}

*Values represented as mean $\pm S D(n=3)$ 


\section{Preparation of CD complexes:}

Various methods as explained below were tried for the preparation of inclusion complex of $\mathrm{RH}$ with $\beta \mathrm{CD}$ or $\mathrm{HP} \beta C D$. The yield as well as drug complexes of the inclusion complexes so prepared were determined by the method described in a subsequent section.

\section{Kneading method ${ }^{[19]}$ :}

$\mathrm{RH}$ and $\beta C D$ or HP $\beta C D$ with molar ratio as given in Table 2 were accurately weighed and transferred to a mortar. The mixture was then triturated in a mortar with a small volume ( $1 \mathrm{ml}$ per $\mathrm{g}$ of mixture) of watermethanol $(1: 1 \mathrm{v} / \mathrm{v})$ solution till a homogenous paste was formed. The paste that formed was kneaded for $30 \mathrm{~min}$ and then dried at $50^{\circ}$ in an oven. The dried mass was passed through a $20 \#$ sieve.

\section{Solvent evaporation method ${ }^{[20]}$ :}

Weighed quantities of $\beta C D$ or HP $\beta C D$ and $R H$ were dissolved in methanol. Evaporation of methanol was done at $65^{\circ}$ under vacuum of $110 \mathrm{~mm} \mathrm{Hg}$ using rotary evaporator to obtain a solid powdered inclusion complex. The complex was scrapped from round bottom flask and passed through $20 \#$ sieve.

\section{Co-precipitation method ${ }^{[21]}$ :}

RH (200 mg) was added to 2:1 water:methanol and the mass so obtained was heated at $55-60^{\circ}$ until complete dissolution. $\beta C D$ and $\mathrm{HP} \beta C D$ as per molar ratio (Table 2 ) were then added. The mass was completely dissolved at $55-60^{\circ}$. The solution was cooled and maintained at $8-10^{\circ}$ to assist precipitation. The precipitates were separated by filtration and dried in hot air oven at $50^{\circ}$.

\section{Freeze drying method ${ }^{[21]}$ :}

$\mathrm{RH}$ and $\beta \mathrm{CD}$ or HP $\beta \mathrm{CD}$ as per molar ratio (Table 2) were dissolved in water and methanol (2:1) mixture. The mixtures were frozen at $-70^{\circ}$ followed by drying by lyophilization (Heto Drywinner DW 1, Denmark).

\section{Evaluation of CD complexes, percent yield:}

In order to select appropriate method for complexation, the prepared CD complexes were evaluated for percent yield and percent drug complexation. The prepared inclusion complexes using different methods mentioned above were weighed using a high precision calibrated balance (Shimadzu, Japan) and the \% yield was calculated using the Eqn. 3, percent yield = total weight of powdered complex obtained/total weight of $\mathrm{RH}$ and CD taken $\times 100$.

\section{Drug content:}

The accurately weighed quantities of prepared complexes were dissolved in water-methanol (9:1) and the amount of $\mathrm{RH}$ present was estimated using

TABLE 2: OPTIMIZATION OF COMPLEXATION METHOD

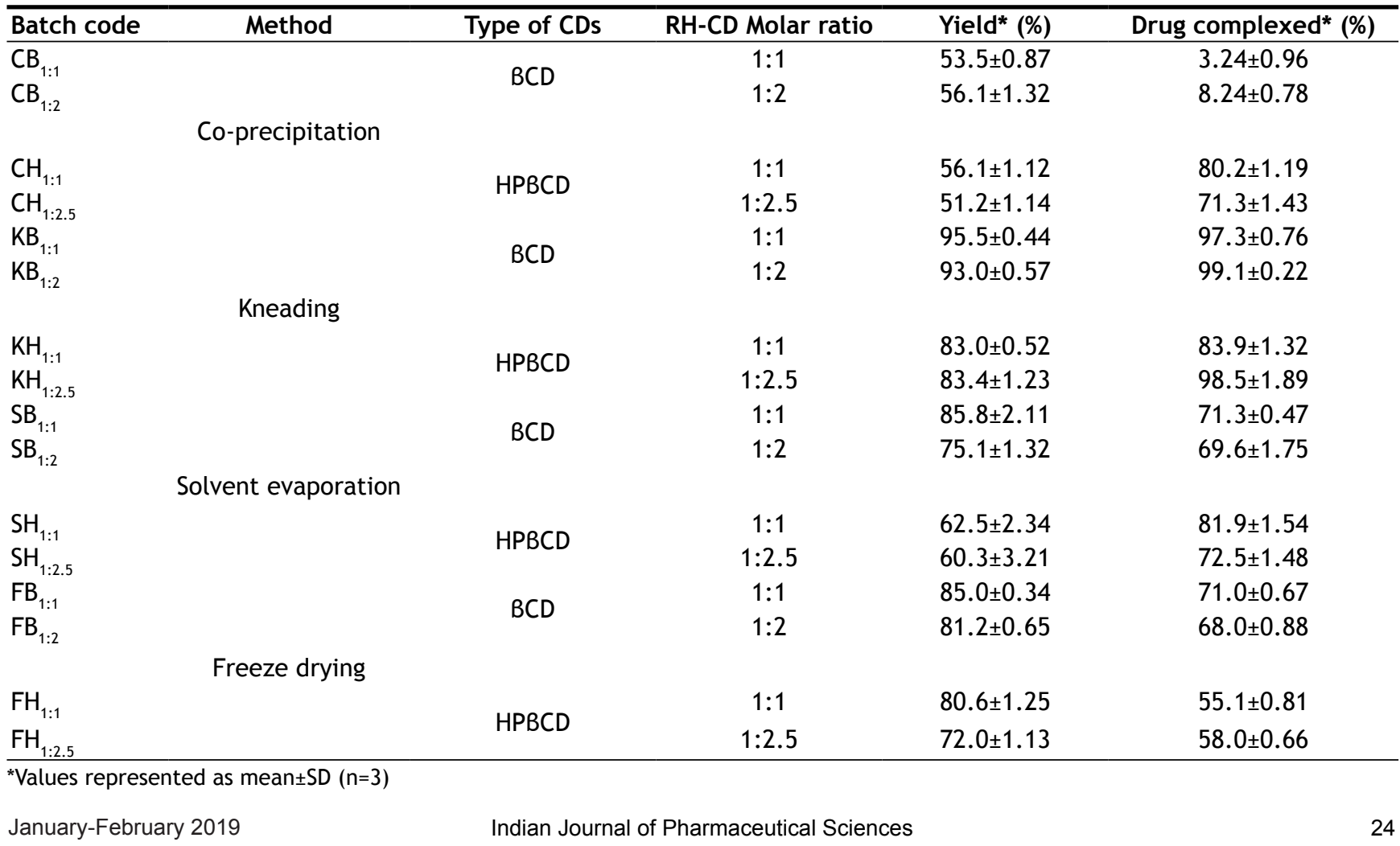


a validated $\mathrm{UV} / \mathrm{Vis}$ spectrophotometric method at $287 \mathrm{~nm} \lambda_{\text {max }}$ to calculate drug content using the Eqn. 4, drug content $=$ amount of RH measured in complexes/ amount of RH initially taken $\times 100$.

\section{Differential scanning calorimetry (DSC):}

DSC analysis was carried out using a differential scanning calorimeter (DSC-60, Shimadzu, Japan) at a heating rate of $10^{\circ}$ per minute in the range of $30-300^{\circ}$ under inert nitrogen atmosphere. DSC thermograms were recorded for $\mathrm{RH}, \mathrm{CDs}$ and their complexes.

\section{Fourier-transform infrared spectroscopy (FTIR):}

FTIR spectroscopy was carried out using potassium bromide disc method. Samples of 1-2 mg each were mixed with potassium bromide, compressed into discs and prepared pellets were scanned using Alpha FTIR spectrophotometer (Bruker Optics, USA) over the range of $4000-500 \mathrm{~cm}^{-1}$. The samples used were $\mathrm{RH}$, CDs and their complexes.

\section{X-ray diffraction (XRD) analysis:}

XRD of RH, CDs, and their complexes were obtained using X'Pert Pro XRD (PANalytical, The Netherlands) equipped with copper high intensity X-ray tube operated at $45 \mathrm{KV}$ and a current of $40 \mathrm{~mA}$ as radiation source. The diffraction patterns were achieved using continuous scan mode with $2 \theta$ values ranging from $5-50^{\circ}$.

\section{Preparation of inclusion complex-loaded hydrogel:}

The optimized CD complex was incorporated into Carbopol 940 hydrogel. The composition of the hydrogel is shown in Table 3. CD complex was added into a mixture of ethanol and propylene glycol and stirred till complete solubilisation. The solution was then added to overnight soaked Carbopol 940 and stirred for uniform mixing. Triethanolamine was added to this dispersion drop-wise and $\mathrm{pH}$ was adjusted to 6.8. This resulted into a transparent hydrogel.

\section{In vitro drug release study:}

In vitro drug release study was performed for the plain drug solution, plain drug hydrogel and CD-loaded hydrogel. Franz diffusion cell was used for the study. Activated dialysis membrane $(12 \mathrm{kD}$, HiMedia Pvt. Ltd, Mumbai) mounted on the Franz diffusion cell was used as permeation barrier. The receptor chamber was filled with $15 \mathrm{ml}$ diffusion medium (phosphate buffer pH 7.4 containing $10 \%$ methanol). The membrane was positioned on the receptor chamber using suitable adhesive. The test formulations containing $0.5 \mathrm{mg}$ $\mathrm{RH}$ were applied to the membrane. The diffusion medium was continuously stirred. Samples $(3 \mathrm{ml})$ were withdrawn from the receptor compartment at predetermined time intervals over $24 \mathrm{~h}$ and the cell was replenished with the same volume of fresh diffusion medium. The samples were analysed using a UV/Vis spectrophotometric method at $287 \mathrm{~nm}$ for estimation of RH.

\section{Ex vivo drug permeation and skin deposition study:}

The samples studied for ex vivo skin permeation and skin deposition studies were plain drug solution, plain drug hydrogel and inclusion complex-loaded hydrogel. The studies were carried out using full thickness pig ear skin. The study for all the above mentioned samples was performed for intact skin as well as skin pre-treated using microneedles. Freshly excised pig ear skin was dipped in hot water and subcutaneous fat was removed using scalpel. It was then impregnated in phosphate buffer ( $\mathrm{pH}$ 7.4)-glycerol (9:1) and preserved at $-70^{\circ}$ for not more than two months. Before starting the experiment, the skin was thawed at room temperature, rinsed with phosphate buffer ( $\mathrm{pH}$ 7.4) and mounted on the Franz diffusion cell. Receptor chamber was filled with $15 \mathrm{ml}$ of 9:1 phosphate buffer ( $\mathrm{pH}$ 7.4):methanol as diffusion medium. The skin was positioned on the receptor chamber with SC facing upwards and fixed using a suitable adhesive. The excess skin was trimmed off and the whole assembly was put on magnetic stirrer. Before applying formulation, skin was placed in diffusion media for $30 \mathrm{~min}$ to achieve equilibration. The test formulations containing $0.5 \mathrm{mg}$ RH were applied to the skin. The content of the cell was continuously stirred at $100 \mathrm{rpm}$. Samples $(3 \mathrm{ml})$ were withdrawn from the receptor compartment at predetermined time intervals over $24 \mathrm{~h}$ and each time an equal volume of fresh diffusion medium was replaced. After suitable dilution, samples were analysed on a UV spectrophotometric method at $287 \mathrm{~nm}$ absorption maximum. At end of

TABLE 3: COMPOSITION OF INCLUSION COMPLEX-LOADED HYDROGELS

\begin{tabular}{lc}
\hline Components & Concentration (\% w/w) \\
\hline Raloxifene $\mathrm{HCl}$ containing & 0.1 \\
complexes & 2 \\
Carbopol 940 & 20 \\
Ethanol & 10 \\
Propylene glycol & 0.07 \\
Triethanolamine & Up to 100 \\
Water & \\
\hline
\end{tabular}


permeation experiments $(24 \mathrm{~h})$, the skin surface was washed three times with the diffusion medium $(15 \mathrm{ml})$ to remove the drug retained on the skin. About $3 \mathrm{ml}$ aliquot from this washing was suitably diluted and analysed by UV spectrophotometric method. The drug deposited within the skin was also estimated for which washed skin was cut into small pieces using a chopper. All pieces were collected in methanol $(20 \mathrm{ml})$ and to extract out the drug present in these pieces, the mix was subjected to bath sonication for 3 cycles each of 5 min followed by homogenization for 5 min under cold condition. The extracted out drug was then separated by centrifugation at $4000 \mathrm{rpm}$ for $10 \mathrm{~min}$. The supernatant was collected and drug present in it was analysed by UV spectrophotometric method as mentioned above.

To study the effect of microneedles pre-treatment on the drug permeation, micropores were created on the skin by microneedle stamp (Dermastamp ${ }^{\circledR}$ ) using our earlier reported method ${ }^{[13]}$. The experiments were performed using the procedure described above.

\section{Histopathological study:}

Optimized $\mathrm{KH}_{1: 2.5}$ hydrogel containing $0.5 \mathrm{mg}$ drug was applied on freshly excised rat abdominal skin. After six hours, skin was immersed in $10 \%$ buffered formalin, dehydrated in gradually increasing concentrations of ethanol, immersed in xylene and finally embedded in paraffin. The 5- $\mu \mathrm{m}$ thick sections of skin were cut from these paraffin blocks using microtome and placed on glass slides. The paraffin wax was removed by gently warming the slide and washing the molten wax with xylene. Sections were then washed with absolute alcohol and water and stained with hematoxylin and eosin to determine gross histopathology and collagen deposition, respectively. Commercial glycerol's mounting fluid was used to finally mount the stained sections. Negative control and positive control slides were also prepared by treating rat skin with phosphate buffer solution $\mathrm{pH} 7.4$ and isopropyl alcohol (IPA) respectively using the method as mentioned above. The slides were analysed at 10-fold magnification using optical microscope (Magnus MLX).

\section{Stability Study:}

A stability study was performed as per ICH guidelines to establish product's integrity with respect to desirable characteristics. The optimized CD complexes hydrogel samples were filled in aluminium tubes, which were sealed and stored under two different environmental conditions viz., $4 \pm 2^{\circ}$ with ambient $\mathrm{RH} ; 30 \pm 2^{\circ}$ with
$60 \pm 5 \%$ RH. Samples were withdrawn from the tube fortnightly for $3 \mathrm{mo}$, diluted with methanol and evaluated for percent drug retained as an indicator of stability.

\section{RESULTS AND DISCUSSION}

Inclusion complexation with CDs has been reported to enhance the solubility of guest molecules which have the ability to fit into the hydrophobic cavity. Out of the various types of $\mathrm{CDs}, \beta C D$ and its derivatives are most widely used in pharmaceutical dosage forms. $\beta C D$ is the most accessible, the lowest-priced and generally the most useful. Derivatives of $\beta C D$ such as HP $\beta C D$ are also preferred for preparation of inclusion complexation because of the higher solubility compared to the parent $\mathrm{CD}$. RH is poorly water soluble drug and hence its solubilisation is necessary prior to its incorporation into a transdermal delivery system. Inclusion complexation with $\beta C D$ and $\mathrm{HP} \beta \mathrm{CD}$ increase in the apparent aqueous solubility of the drug and thereby amplify the thermodynamic driving force for transdermal permeation. Additionally, high concentrations of CDs can disrupt the protein structure of the SC promoting the transdermal permeation of solubilized compounds ${ }^{[22]}$.

Phase solubility study is useful for evaluation of the affinity between $\beta C D$, the host molecule and guest molecule. The typical phase solubility diagrams observed for different types of drugs is shown in fig. 1a and the phase solubility diagram obtained for $\mathrm{RH}$ inclusion complex with $\beta C D$ and HP $\beta C D$ is shown in fig. 1b. As shown in fig. 1a, the phase solubility diagram falls into two main categories, A- and B-type. A-type curves indicative for the formation of soluble inclusion complexes, while B-type behaviour is suggestive of the formation of inclusion complexes of poor solubility. The phase solubility diagram of $\mathrm{RH}$ with $\beta C D$ is shown in fig. $1 \mathrm{~b}$. It is evident that the aqueous solubility of RH increases linearly as a function of $\beta C D$ concentration up to $1: 2$ molar ratio. With further increase in the concentration of $\beta C D$, there was a decrease in the solubility. The phase solubility diagram is of Bs type, which indicates formation of a complex with limited solubility. The stability constant $\left(\mathrm{K}_{1: 1}\right)$ obtained with $\beta \mathrm{CD}$ was $1572 \mathrm{M}^{-1}$, indicating formation of stable 1:1 molar ratio complex. Highest solubility was obtained with 1:2 molar ratio. So, for preparation of inclusion complex with $\beta C D$, two different molar ratios viz. 1:1 and 1:2 were selected. 

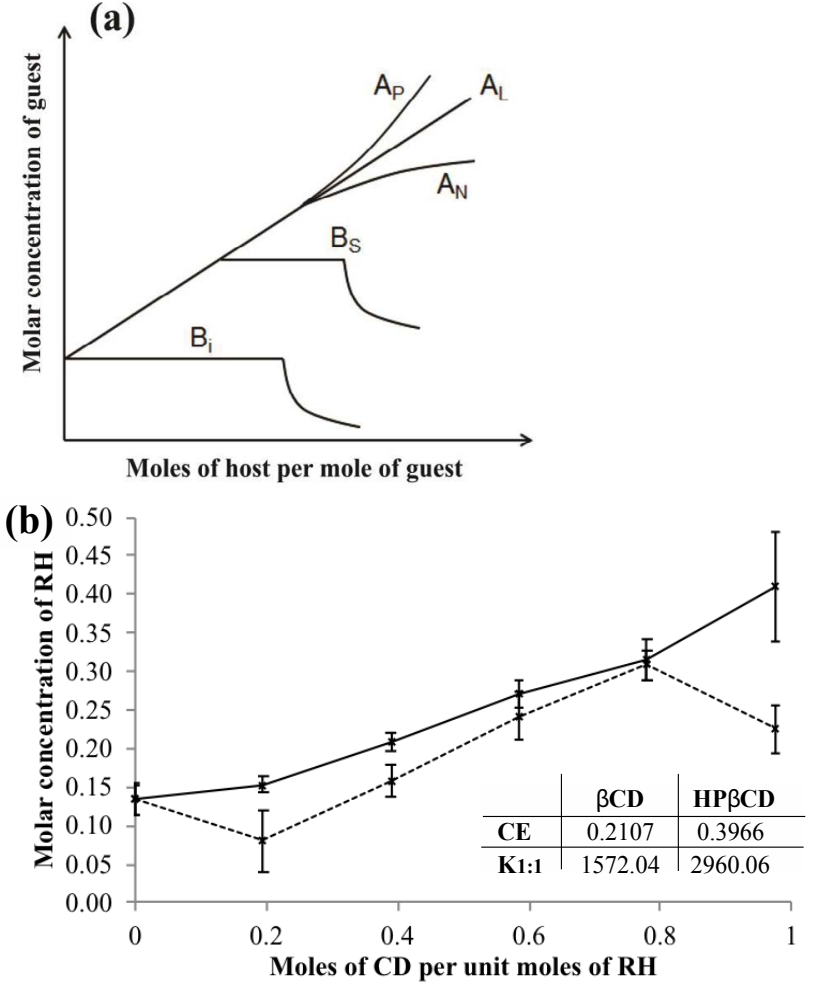

Fig. 1: Phase solubility curves

Phase solubility curves of (a) typical phase solubility curves reported for different types of drugs and (b) phase solubility curves obtained for $\mathrm{RH}$ inclusion complex with $\beta C D$ and HPßCD. -- $\times--$ Complexed with $\beta C D,-\times-$ complexed with HPßCD

The CE was found to be 0.210 . The phase solubility diagram of the RH with HP $\beta C D$ is shown in fig. $1 \mathrm{~b}$. The aqueous solubility of RH increases linearly as a function of HP $\beta C D$ concentration. Phase solubility diagram of $\mathrm{RH}$ in the presence of $\mathrm{HP} \beta \mathrm{CD}$ can be classified as the Ap type indicating formation of higher order complexes with respect to the ligand at higher ligand concentrations. Stability constant with HP $\beta C D$ was obtained $2960 \mathrm{M}^{-1}$, indicates formation of stable 1:1 molar ratio complex and from highest solubility was obtained with 1:2.5 molar ratio. The CE calculated was 0.397 . The significantly higher values of stability constant and the CE in case of HP $\beta C D$ compared to the $\beta C D$ indicates that more stable complex with higher efficiency is obtained with HP $\beta C D$. It has been reported that while $\beta C D$ often gives rise to B-type curves due to the poor water solubility of the ligand itself, the chemically modified CDs including HP $\beta C D$ and SBE $\beta C D$ usually produce soluble complexes (i.e. A-type systems).

Various methods were tried for the preparation of the inclusion complex using the drug:ligand ratios selected in phase solubility studies. The \% yield and $\%$ drug complexes of the different batches are shown in Table 2. It is evident that the highest yield and $\%$ drug complexes were obtained using kneading method while lowest values were obtained by co-precipitation method. The reason for lower yield may be the incomplete precipitation at the cooling temperature. Co-precipitation method has not received considerable success owing to low yield, risk of using organic solvents, and longer time required for the preparation in larger scale ${ }^{[23]}$. Kneading method is simpler and does not require sophisticated instrumentation and hence it is more feasible at larger scale. Thus further investigations were done on the batches prepared using kneading method.

Fig. $2 \mathrm{a}$ and $2 \mathrm{~b}$ showed the DSC thermograms of pure components and their corresponding complexes, $\mathrm{RH}-$ $\beta C D$ and $\mathrm{RH}-\mathrm{HP} \beta \mathrm{CD}$, respectively. DSC thermogram of RH showed two endotherms, one at $110^{\circ}$ and another at $270^{\circ}$. The endotherm at $110^{\circ}$ could be attributed to the loss of water of hydration, while the sharp endotherm at $270^{\circ}$ corresponds to the melting point of RH. However, the thermogram of $\beta C D$ and HP $\beta C D$ showed a broad and weak endothermic effect, which attained a maximum around $108^{\circ}$ and $84^{\circ}$, respectively. These results might be due to the release of water ${ }^{[24]}$. The DSC thermograms of inclusion complex of $\mathrm{RH}$ with $\beta C D$ show an endotherm at $259^{\circ}$ indicating noncomplexation or incomplete complexation of $\mathrm{RH}$ with $\beta C D$. The Bs type of curve obtained in the phase solubility studies support this finding. However, in case of the inclusion complexation with $\mathrm{HP} \beta \mathrm{CD}$, there was a complete disappearance of the RH endothermic peak for both the ratios viz. 1:1 and 1:2.5. This could be attributed to the formation of an amorphous solid dispersion, and/or the molecular encapsulation of drug inside the CD cavity ${ }^{[24]}$. These results suggested the formation of inclusion complexes between HP $\beta C D$ and RH.

Fig. $3 \mathrm{a}$ and $3 \mathrm{~b}$ showed the FTIR spectra of $\mathrm{RH}, \beta C D$, HP $\beta C D$ and their inclusion complexes. FTIR spectrum of $\mathrm{RH}$ is characterized by principal absorption peaks at $1642 \mathrm{~cm}^{-1}$ (C=0 group stretching), $1596 \mathrm{~cm}^{-1}$ (C-O-C group stretching), $1465 \mathrm{~cm}^{-1}$ (S-benzothiofuran ring). In $\beta C D$ FTIR spectrum, peak at $3360 \mathrm{~cm}^{-1}$ was due to free $-\mathrm{OH}$ group vibration. The FTIR spectrum of $\mathrm{HP} \beta \mathrm{CD}$ shows peaks at $3384 \mathrm{~cm}^{-1}$ (-OH group vibration) and around $3800 \mathrm{~cm}^{-1}(\mathrm{C}=\mathrm{O}$ glucosidic group stretching). FTIR spectrum of RH with $\beta C D$ and $\mathrm{HP} \beta C D$ showed significantly reduced intensity and slight shifting of characteristic peak of drug. Such 

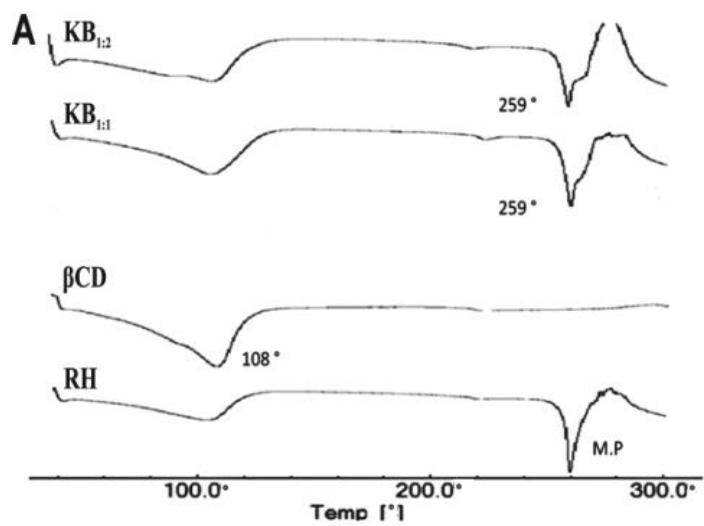

B
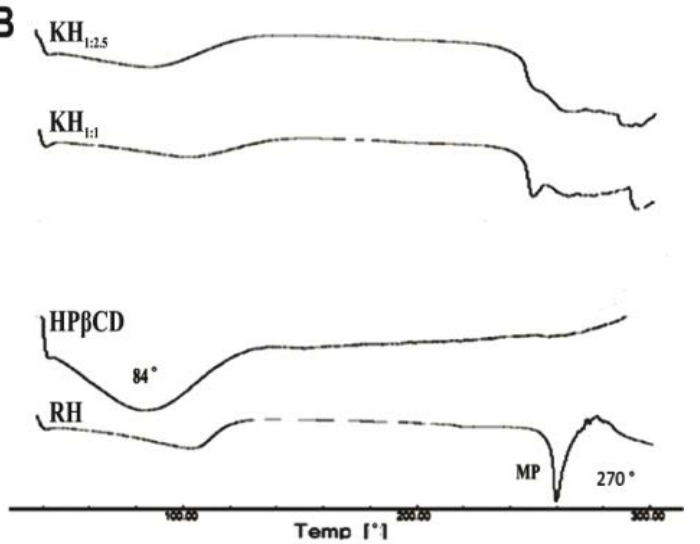

Fig. 2: DSC thermograms

(A) RH, $\beta C D$ and their complexes; (B) RH, HPBCD and their complexes

results could be attributed to the complex formation between RH and CDs. The XRD pattern of RH showed (fig. 4a and $4 \mathrm{~b}$ ) intense and sharp peaks, indicating its crystalline nature. Crystallinity was determined by comparing some representative peak heights in the diffraction patterns of the complexes with those of a reference (pure RH). The peak intensities of pure $\mathrm{RH}$ and its corresponding complexes are presented in Table 4. In the RH powder XRD, intense peaks at a diffraction angle $(\theta)$ of $2^{\circ}$, at position of $14.5,15.8$ and 22.7 depicts the crystalline nature of the drug. Similarly, diffractograms of $\beta C D$ exhibited a series of intense peaks, showing its crystalline character. However, the XRD pattern of the RH- $\beta$ CD of molar ratio 1:1 showed fewer, broader, and less intense peaks and molar ratio 1:2 showed a further decrease in peak area of drug (fig. 4a and 4b). At diffraction angle $(\theta)$ of $2^{\circ}$, at position of 15.8 , area of complex was decreased than area of $\beta C D$. This reduction in crystallinity is an indication of inclusion complex formation which was better in 1:2 molar ratio compared to $1: 1$. The absence of any peak in the HP $\beta C D$ diffractograms revealed the amorphous nature of this compound. XRD pattern of RH-HP $\beta C D$ complexes, molar ratio $1: 1$ and 1:2.5 showed a reduction in peak intensity. These results suggested increased amorphous nature of drug due to inclusion complex formation.

The release profile of $\mathrm{RH}$ solution, plain $\mathrm{RH}$ hydrogel and different formulations is shown in fig. 5. It is evident that the drug release after $24 \mathrm{~h}$ from the plain drug dispersion was $35.3 \%$, which was slightly higher than that obtained from the plain drug hydrogel. This might be due to the viscosity effect imparted by the gelling component. In case of inclusion complex-loaded hydrogels, both $\beta C D$ and $\mathrm{HP} \beta C D$ showed significantly higher drug release than the plain drug indicating the solubilizing effect of the CDs. However, the effect was more pronounced for HP $\beta C D$ with highest drug release obtained for the ratio $1: 2.5$. This finding is supported by the phase solubility studies and DSC studies,
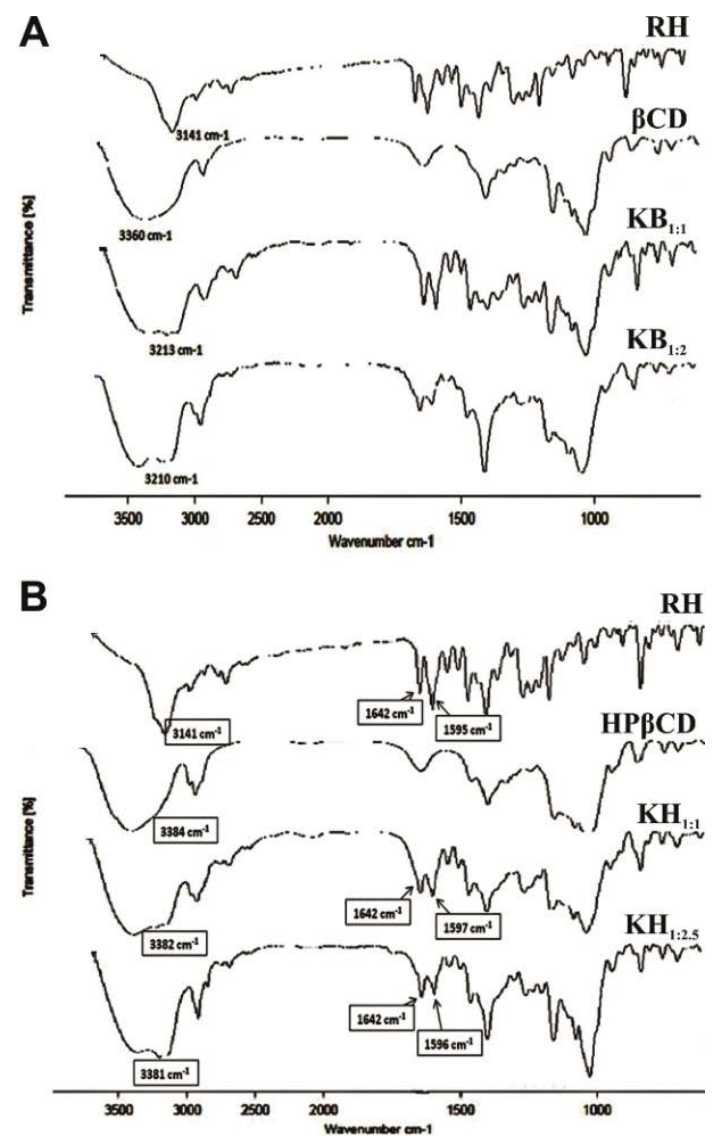

Fig. 3: FTIR spectra

(A) RH, $\beta C D$ and their complexes; (B) RH, HPBCD and their complexes

TABLE 4: XRD PEAK AREAS OF RH, $\beta C D, H P \beta C D$ AND THEIR COMPLEX

\begin{tabular}{lcccccc}
\hline $\begin{array}{l}\text { Position } \\
{\left[{ }^{\circ} \text { 2 } \theta\right]}\end{array}$ & RH & $B C D$ & $\mathrm{~KB}_{1: 1}$ & $\mathrm{~KB}_{1: 2}$ & $\mathrm{KH}_{1: 1}$ & $\mathrm{KH}_{1: 2.5}$ \\
\hline 14.5 & 253.33 & 73.84 & 165.12 & 91.77 & 92.9 & 21.11 \\
15.8 & 159.75 & 191.11 & 81.73 & 50.69 & 59.9 & 21.07 \\
22.7 & 544.35 & 271.19 & 275.51 & 237.65 & 172.8 & 40.87 \\
\hline
\end{tabular}


A
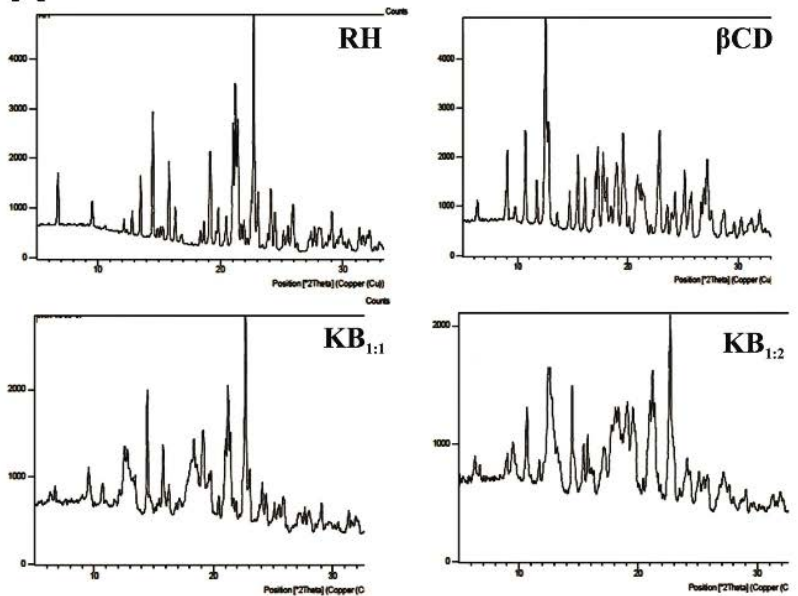

B
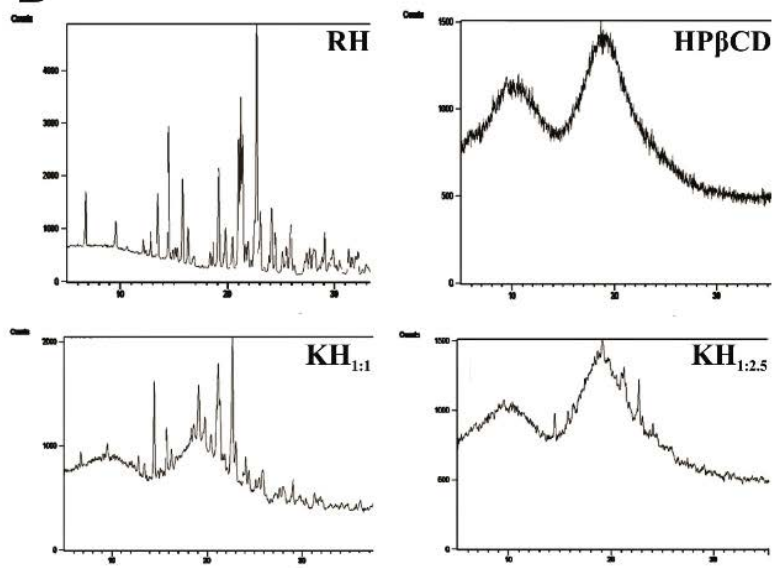

Fig. 4: X-ray diffractograms (A) RH, $\beta C D$ and their complexes; (B) RH, HPßCD and their complexes

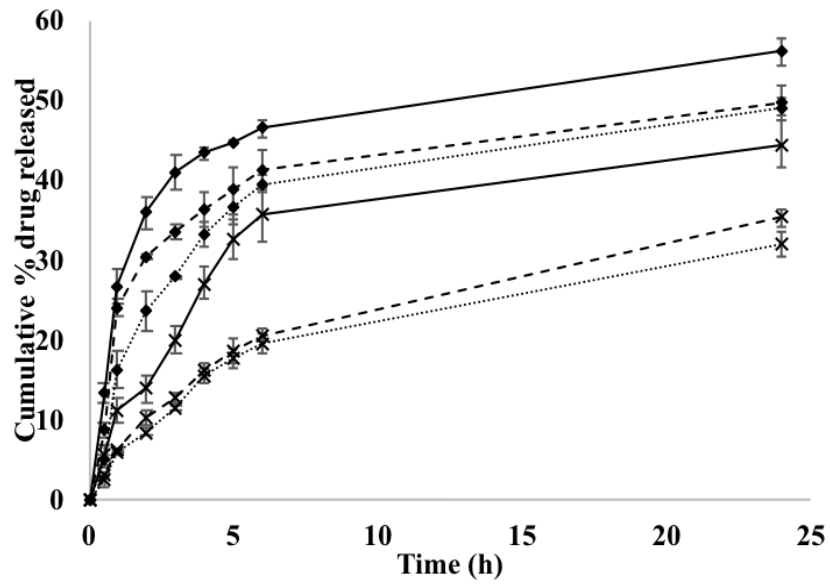

Fig. 5: In vitro drug release profile

In vitro drug release profile of different formulations via dialysis bag (MWCO, $12 \mathrm{kD})$; --×-- drug solution, ... $\times$... plain gel, ... ... RH:HPßCD (1:1), — — RH:HPßCD (1:2.5), —×-

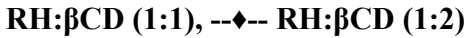

which indicated the superiority of HPßCD in forming inclusion complex with RH.

The results of the ex vivo studies conducted on full thickness pig ear skin are shown in Table 5. There was a good correlation between the results of the in vitro drug release studies and the ex vivo drug permeation studies. The results indicated that inclusion complex formed with $\mathrm{HP} \beta C D$ at a ratio of 1:2.5 showed maximum drug permeation after $24 \mathrm{~h}$. This might be due to the presence of higher amount of $\mathrm{CD}$, which is a known penetration enhancer along with the high solubilisation of RH due to inclusion complexation. The highest permeation through intact skin obtained was around $43 \%$. In order to further enhance the permeation, the skin was pre-treated with microneedles and this significantly increased the permeation to around $58 \%$. Microneedles are able to create micropores in the skin without causing pain to the patient as they have very short length and small diameter. Fig. 6 shows the $\%$ drug retained on the surface, $\%$ drug deposited within the skin and the $\%$ drug release from different samples. It can be seen that highest $\%$ of drug retained on the surface was for plain drug solution followed by the plain drug hydrogel, which indicates the very less ability of the plain drug to cross the SC layer. Microporation led to a decrease in the drug retained on the skin surface indicating the effect of creation of pores on enhancement of drug permeation. Highest skin deposition $(13.8 \pm 1.29 \%)$ was found with $\mathrm{KH}_{1.2 .5}$ hydrogel due to more carrying capacity of RH-HP $\beta C D$ complex. This further increased the skin deposition to $15.6 \%$. Thus, the inclusion complex formed using HP $\beta C D$ at a molar ratio of 1:2.5 (RH:HP $\beta C D$ ) was found to give maximum transdermal permeation, which was significantly increased by microporation of the skin.

Histopathology studies were thus conducted on this optimized formulation to visualize the pores and assess any toxic or irritation potential of the formulation. The images obtained in these studies are shown in fig. 7. It was found that IPA-treated rat skin showed disruption and swelling in the epidermal and subepidermal regions, which was absent in the skin treated with phosphate buffer solution $\mathrm{pH}$ 7.4. $\mathrm{KH}_{1: 2.5}$ hydrogel contained HP $\beta C D$ and Carbopol 940, which are nontoxic to skin. $\mathrm{KH}_{1: 2.5}$ hydrogel showed insignificant disruption, damage and oedema in the epidermal and sub epidermal regions. That was indication of nontoxicity of formulation on rat skin. The results of the stability studies are shown in Table 6. It is evident that the prepared formulation is more stable under refrigerated conditions than at room temperature.

$\mathrm{RH}$ could form a stable inclusion complex with HP $\beta C D$. Out of the different methods tried, kneading method 
TABLE 5: EX VIVO DRUG PERMEATION STUDY

\begin{tabular}{|c|c|c|c|c|c|c|c|c|}
\hline \multirow[b]{2}{*}{ Time (h) } & \multicolumn{6}{|c|}{ Intact skin* } & \multicolumn{2}{|c|}{ Microporated skin* } \\
\hline & $\begin{array}{c}\text { Plain } \\
\text { drug solution }\end{array}$ & $\begin{array}{c}\text { Plain drug } \\
\text { hydrogel }\end{array}$ & $\begin{array}{c}\mathrm{KB}_{1: 1} \\
\text { hydrogel }\end{array}$ & $\begin{array}{c}\mathrm{KB}_{1: 2} \\
\text { hydrogel }\end{array}$ & $\begin{array}{c}\mathrm{KH}_{1: 1} \\
\text { hydrogel }\end{array}$ & $\begin{array}{c}\mathrm{KH}_{1: 2.5} \\
\text { hydrogel }\end{array}$ & $\begin{array}{c}\text { Plain drug } \\
\text { hydrogel }\end{array}$ & $\begin{array}{c}\mathrm{KH}_{1: 2.5} \\
\text { hydrogel }\end{array}$ \\
\hline 0 & 0 & 0 & 0 & 0 & 0 & 0 & 0 & 0 \\
\hline 0.5 & $0.5 \pm 0.03$ & $0.4 \pm 0.02$ & $0.8 \pm 0.04$ & $2.4 \pm 0.06$ & $0.8 \pm 0.06$ & $4.8 \pm 0.03$ & $0.7 \pm 0.04$ & $6.3 \pm 0.22$ \\
\hline 1 & $1.9 \pm 0.10$ & $1.6 \pm 0.09$ & $4.6 \pm 0.47$ & $5.5 \pm 0.17$ & $2.5 \pm 0.44$ & $6.3 \pm 0.25$ & $2.1 \pm 0.42$ & $9.5 \pm 0.47$ \\
\hline 2 & $5.4 \pm 0.17$ & $5.7 \pm 0.23$ & $6.2 \pm 0.87$ & $6.7 \pm 0.21$ & $5.1 \pm 0.67$ & $9.4 \pm 0.43$ & $3.8 \pm 0.76$ & $11.7 \pm 0.76$ \\
\hline 3 & $6.8 \pm 0.24$ & $8.4 \pm 0.41$ & $10 \pm 0.75$ & $9.6 \pm 0.32$ & $6.3 \pm 1.56$ & $11.6 \pm 0.54$ & $4.9 \pm 0.98$ & $16.8 \pm 0.97$ \\
\hline 4 & $8.1 \pm 0.47$ & $10.1 \pm 0.54$ & $11.8 \pm 0.92$ & $11.3 \pm 0.54$ & $9.4 \pm 0.78$ & $14.1 \pm 1.26$ & $7.9 \pm 1.12$ & $20.6 \pm 1.09$ \\
\hline 5 & $8.9 \pm 0.39$ & $11.2 \pm 0.68$ & $13.6 \pm 0.87$ & $13.8 \pm 1.26$ & $11.6 \pm 1.46$ & $16.2 \pm 1.23$ & $11.7 \pm 1.35$ & $23.7 \pm 1.43$ \\
\hline 6 & $9.4 \pm 0.61$ & $12.3 \pm 1.12$ & $14.6 \pm 1.43$ & $14.7 \pm 1.75$ & $12.6 \pm 1.34$ & $19.3 \pm 1.87$ & $17.8 \pm 1.32$ & $30.7 \pm 1.54$ \\
\hline 24 & $18.3 \pm 1.01$ & $22.8 \pm 1.06$ & $26.3 \pm 1.47$ & $29.8 \pm 1.89$ & $28.5 \pm 0.98$ & $42.9 \pm 1.34$ & $31.7 \pm 1.55$ & $58.4 \pm 1.87$ \\
\hline $\mathrm{J}_{\mathrm{SS}}^{\#}$ & 1.6 & 2.581 & 2.876 & 3.908 & 3.711 & 5.801 & 3.407 & 6.809 \\
\hline PER & - & 1.61 & 1.80 & 2.44 & 2.32 & 3.63 & 2.13 & 4.26 \\
\hline
\end{tabular}

*Values represented as mean $\pm \mathrm{SD}(\mathrm{n}=3)$; ${ }^{*}$ in $\mu \mathrm{g} / \mathrm{cm}^{2} / \mathrm{h}$

TABLE 6: STABILITY STUDY OF $\mathrm{KH}_{1: 2.5}$ HYDROGEL

\begin{tabular}{lcc}
\hline \multirow{2}{*}{$\begin{array}{l}\text { Time } \\
\text { (months) }\end{array}$} & \multicolumn{2}{c}{ Drug retained* $(\%)$} \\
\cline { 2 - 3 } & $\begin{array}{c}\mathbf{4} \pm 2^{\circ} \\
\text { ambient } \mathrm{RH}\end{array}$ & $\begin{array}{c}30 \pm 2^{\circ} \\
\mathbf{6 0 \pm 5} \% \mathrm{RH}\end{array}$ \\
\hline 0 & $100.0 \pm 0.00$ & $100.0 \pm 0.00$ \\
1 & $100.0 \pm 0.00$ & $98.2 \pm 0.03$ \\
2 & $99.1 \pm 0.42$ & $95.0 \pm 0.51$ \\
3 & $96.6 \pm 0.61$ & $94.7 \pm 0.32$ \\
\hline
\end{tabular}

*Values represented as mean $\pm S D(n=3)$

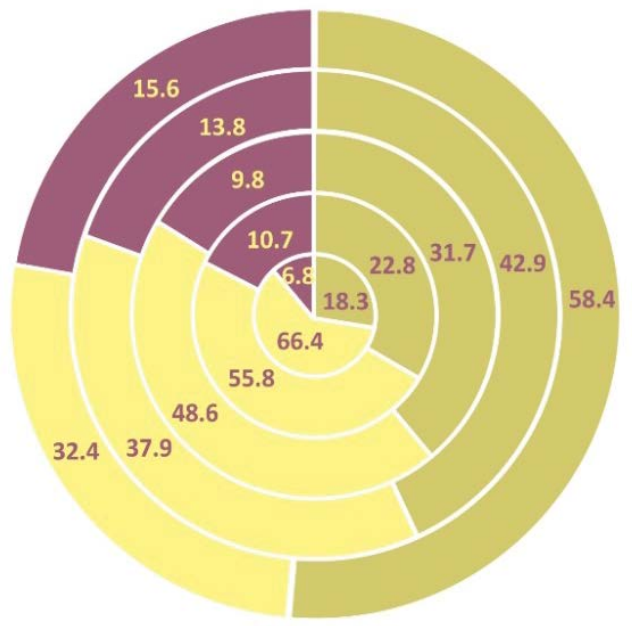

Fig. 6: Ex vivo drug retention, deposition and permeation profile Ex vivo drug retention, deposition and permeation profile of different formulations via full thickness pig ear skin. From center to surface: circle 1- plain drug solution through intact skin, circle 2- plain hydrogel though intact skin, circle 3plain hydrogel through microporated skin, circle 4- KH 1:2.5 hydrogel though intact skin, circle 5- KH 1:2.5 hydrogel though microporated skin. — Drug deposited within the skin (\%), drug permeated across the skin $(\%)$, $\square$ Drug retained on the skin surface $(\%)$

was found to give maximum yield and drug complexed. In vitro drug release studies indicated a significantly higher release from the inclusion complexes than the plain drug. Ex vivo studies revealed enhancement of

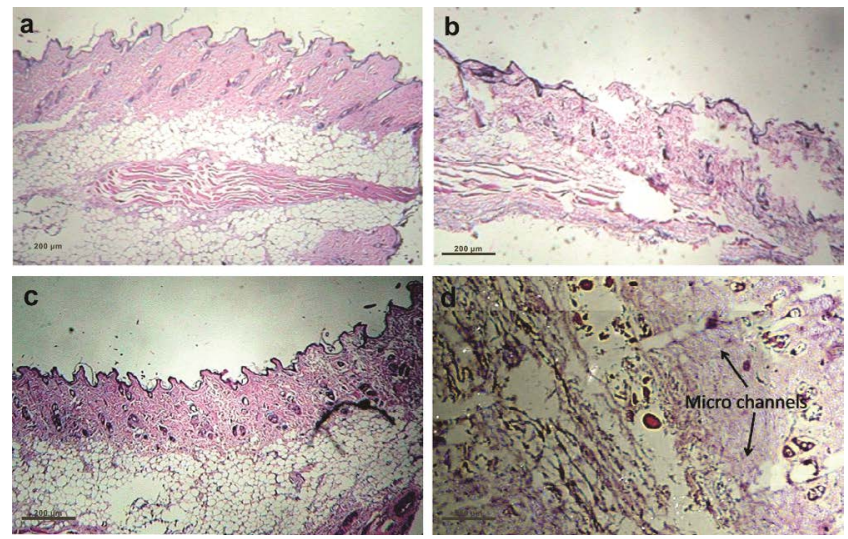

Fig. 7: Microscopic photographs of histological sections Photomicrographs of histological sections of rat abdominal skin treated with (a) phosphate buffer saline (pH 7.4), (b) isopropyl alcohol, (c) KH1:2.5 hydrogel and (d) DermaStamp ${ }^{\circledR}$ microneedle

transdermal permeation with the use of inclusion complexation, which could be further increased upon pre-treatment of skin with microneedles. The histopathology studies indicated a non-irritating nature of the developed formulation with clear visualization of the formation of micropores. Extended research involving pharmacokinetic and pharmacodynamic studies may be able to prove the superiority of the formulation upon the marketed tablet dosage form.

\section{Acknowledgements:}

Authors express thanks to Aarti Drugs Ltd., Mumbai, India and Polar Genetics, Jalandhar, India for providing gift samples of Raloxifene hydrochloride and pig ears, respectively.

\section{Conflict of interest:}

There is no conflict of interest. 


\section{Financial support and sponsorship:}

Nil.

\section{REFERENCES}

1. Couse JF, Korach KS. Estrogen receptor null mice: what have we learned and where will they lead us? Endocr Rev 1999;20:358-417.

2. Chen FP, Hsu T, Hu CH, Wang WD, Wang KC, Teng LF. Expression of estrogen receptors alfa and beta mRNA and alkaline phosphatase in the differentiation of osteoblasts from elderly postmenopausal women: comparison with osteoblasts from osteosarcoma cell lines. Taiwan J Obstet Gynecol 2006;45:307-12.

3. Riggs BL, Khosla S, Melton LJ 3rd. Sex steroids and the construction and conservation of the adult skeleton. Endocr Rev 2002;23:279-302.

4. Zs S. Estrogen Prevention for Breast Cancer. 1st ed. Hauppauge, New York, United States: Nova Science Publishers, Inc.; 2013. p. 1-22.

5. Beral V; Million Women Study Collaborators. Breast cancer and hormone-replacement therapy in the Million Women Study. Lancet 2003;362:419-27.

6. Rossouw JE, Anderson GL, Prentice RL, LaCroix AZ, Kooperberg C, Stefanick ML, et al. Risks and benefits of estrogen plus progestin in healthy postmenopausal women: principal results From the Women's Health Initiative randomized controlled trial. JAMA 2002;288:321-33.

7. Mitlak BH, Cohen FJ. In search of optimal long-term female hormone replacement: the potential of selective estrogen receptor modulators. Horm Res 1997;48:155-63.

8. Teeter JS, Meyerhoff RD. Environmental fate and chemistry of raloxifene hydrochloride. Environ Toxicol Chem 2002;21:729-36.

9. Thakkar H, Nangesh J, Parmar M, Patel D. Formulation and characterization of lipid-based drug delivery system of raloxifene-microemulsion and self-microemulsifying drug delivery system. J Pharm Bioallied Sci 2011;3:442-8.

10. Patel BD, Modi RV, Thakkar NA, Patel AA, Thakkar PH. Development and characterization of solid lipid nanoparticles for enhancement of oral bioavailability of Raloxifene. J Pharm Bioallied Sci 2012;4:S14-S16.

11. Wempe MF, Wacher VJ, Ruble KM, Ramsey MG, Edgar $\mathrm{KJ}$, Buchanan NL, et al. Pharmacokinetics of raloxifene in male Wistar-Hannover rats: Influence of complexation with hydroxybutenyl-beta-cyclodextrin. Int $\mathrm{J}$ Pharm 2008;346:25-37.

12. Mahmood S, Taher M, Mandal UK. Experimental design and optimization of raloxifene hydrochloride loaded nanotransfersomes for transdermal application. Int $\mathrm{J}$ Nanomedicine 2014;9:4331-46.

13. Thakkar HP, Savsani H, Kumar P. Ethosomal Hydrogel of RaloxifeneHCl: Statistical optimization and ex vivo permeability evaluation across microporated pig ear skin. Curr Drug Deliv 2016;13:1111-22.

14. Chauhan BM, Bajpai M. Formulation and evaluation of transdermal drug delivery of raloxifene hydrochloride. Int $\mathrm{J}$ Pharm Sci Res 2010;1:72-9.

15. Reimão JQ, Miguel DC, Taniwaki NN, Trinconi CT, Yokoyama-Yasunaka JK, Uliana SR. Antileishmanial Activity of the Estrogen Receptor Modulator Raloxifene. PLoS Negl Trop Dis 2014;8:e2842.

16. Challa R, Ahuja A, Ali J, Khar RK. Cyclodextrins in drug delivery: an updated review. AAPS PharmSciTech 2005;6:E329-57.

17. Choy YB, Prausnitz MR. The Rule of Five for Non-Oral Routes of Drug Delivery: Ophthalmic, Inhalation and Transdermal. Pharm Res 2011;28:943-8.

18. Higuchi T, Connors K. Phase solubility techniques. Adv Anal Chem Instrum 1965;4:117-212.

19. Hussein A, Ibrahim M, Amin M, Ahmed O, Afouna M. Improved in vitro dissolution parameters and in vivo hypolipidemic efficiency of atorvastatin calcium through the formation of hydrophilic inclusion complex with cyclodextrins. Drug Dev Res 2011;72:379-90.

20. Agrawal R, Gupta V. Cyclodextrins - A Review on Pharmaceutical Application for Drug Delivery. IJPFR 2012;2:95-112.

21. Vianna R, Bentley M, Ribeiro G, Carvalho F, Neto A, de Oliveira D, et al. Formation of cyclodextrin inclusion complexes with corticosteroids: their characterization and stability. Int J Pharm 1998;167:205-13.

22. Kear CL, Yang J, Godwin DA, Felton LA. Investigation into the Mechanism by Which Cyclodextrins Influence Transdermal Drug Delivery. Drug Dev Ind Pharm 2008;34:692-7.

23. Shah N, Seth AK, Balaraman R. Bioavailability enhancement of poorly soluble Raloxifene by Designing Inclusion Complex with $\beta$-Cyclodextrin. Int J Pharm Pharm Sci 2015;7:205-11.

24. Fernandes CM, Vieira TM, Veiga FJ. Physicochemical characterization and in vitro dissolution behaviour of nicardipine-cyclodextrins inclusion compounds. Eur J Pharm Sci 2002;15:79-88 